\title{
A Smart Mobile Application for Assisting Parents in Anti-Drug Support
}

\author{
Tsz Hei Yeung, Chi Kit Ng, Vincent Ng* \\ Department of Computing, The Hong Kong Polytechnic University, Hong Kong, China
}

\begin{tabular}{l} 
A R T I C L E I N F O \\
\hline Article history: \\
Received: 01 December, 2017 \\
Accepted: 15 January, 2018 \\
Online: 10 February, 2018 \\
\hline Keywords: \\
Drug Abuse \\
Mobile Application \\
Location Check-in \\
Behaviour analysis \\
Instant Messaging \\
\hline
\end{tabular}

\begin{abstract}
A B S T R A C T
Drug abuse problem is not a new problem, but it is still serious in Hong Kong. The emergence of hidden drug abuse youth has brought more difficulties to them and their parents. The usual ways of providing anti-drug abuse assistance are not sufficient to these types of young people. Intelligent methods have been developed to assist anti-drug workers. In this paper, we present three mobile applications with a set of intelligent tools for parents and social workers to help our young people. The applications have an alerting tool to detect the possibility of drug abuse of a young student, and to alert corresponding parents and social workers for remedial actions. Another function is the nearby notification tool to help social workers in identifying people who may need assistance. For the alerting function, a number of data mining and text analysis techniques have been adopted for behavior analysis. A supporting instant messaging has been co-developed for communicating alerting messages.
\end{abstract}

\section{Introduction}

Drug abuse problem is not a new problem, but it is still a big problem in Hong Kong Society. Even there is a tendency of decreasing in drug abusing, the government report indicated that the age of first drug taking is decreasing. This shows that the problem is in serious with depth (age) but not the width(quantity). Besides, from the perspective of teenagers, they do not have much knowledge on how to get the treatment service from different organizations.

Furthermore, Hong Kong has another problem which is the hidden youths or "socially withdrawal". The term "hidden youths" is originated from Japanese word "Hikikomori" and it means that a person is scheduled at home by at least six months [1]. There is one more level under the "hidden youths" which is "Otaku" in terms of Japanese. There are different characteristics for "Otaku", but the commonly use is that "Otaku" always stay at home for activities and avoid social activities. A study found that these people are satisfied with their quality of life [2]. Also, it is difficult for other people to interact with them normally. And once they are drug abuser, they become the riskiest group of people as people cannot discover them easily and help them return to life. In Hong Kong, it has been reported that, since 2010, 80\% young drug abusers take drugs at home.

\footnotetext{
*Vincent Ng, Department of Computing, The Hong Kong Polytechnic
}

University, 852-2766-7242, Email: cstyng@,comp.polyu.edu.hk
According to Narcotics Division, the median drug abuse history of newly reported abusers (i.e. the time for abusers to be reported to the CRDA by reporting agencies from their first drug abuse) remained between 1.7 and 2.1 years during 2007 to 2009. It then increased continuously to 5.9 years in 2015 , and dropped to 4.6 years in 2016 [3]. One reason is because psychotropic substance abuse is more popular among young drug abusers [4]. Unlike traditional drugs such as heroin, psychotropic substance abuse is more "hidden" in nature. Young drug abusers tend to take psychotropic substance at home. It is hard for us to bring the young drug abusers to light.

Smartphones have become popular among young people. There are close to 5 million Hong Kong persons aged 10 and over had smartphones in 2014 and the smartphone penetration rate has exceeded $77.2 \%$ [5]. Developing anti-drug mobile applications can be an effective way to pass healthy information to young people as well as parents and social workers. Hence, in 2014, we submitted a proposal to the Beat Drugs Fund in HKSAR to develop a series of mobile applications for students, parents and social workers with a backend platform to support information sharing.

In our design, we aim to find out the hidden or potential drug abusers, and to warn their family and anti-drug workers about the problem. Another important function is to support the nearby function for social workers to access young people. For the rest of the paper, Section 2 provides the background review. Next, in Section 3 and 4, we describe our application design and 
implementation approach. Section 5 concludes our present work in this paper.

\section{Background Review}

\subsection{Anti-Drugs and Behavior Analysis Mobile Apps}

Dealing with the drug abuse problem, different parties in Hong Kong society have developed different mobile applications to link with the youths. There are two mobile applications recently developed in HK and they are "Anti-Drug Information Center" and “毒庱無可忍” to deal with the drug abuse problem. The “Anti-drug Information Center” (禁毒資訊站) developed in Macau provides the drug information as shown in Figure 1 and can support parents as a simple drug abuse detection tool [6].

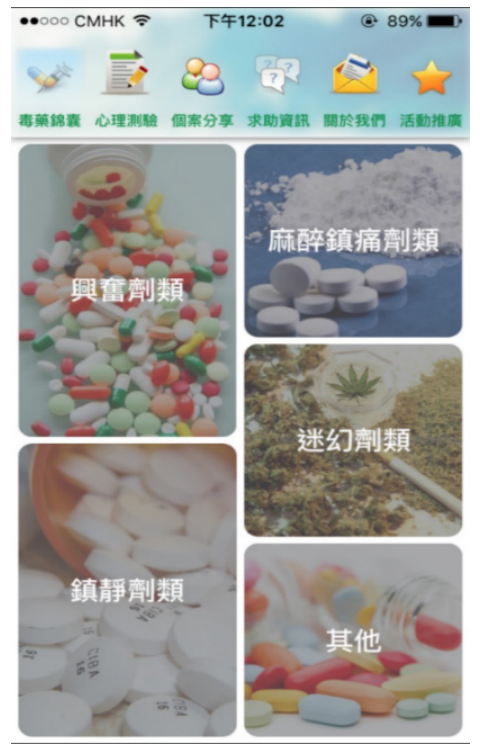

Figure 1. Home screen of the Anti-drug Information Center

This mobile application adopts an expert system approach which is based on the anti-drug resource kit designed by the T.W.G.Hs. CROSS Centre [7]. However, there were criticisms on the test because the behavior criteria could have different interpretations, not only for detecting drug abuse issues. For example, it simply identifies " emotionally unstable" and "performance mysterious contact with friends" as symptoms of drug abuse, but these symptoms can also be characterized adolescent.

For the "毒瘾無可忍” application, it was developed by a Christian organization. It also provides the drug related information and detection test. The information it provided are the real cases sharing, information of referral center and some encouragement message from the bible. The detection test is rulebased and has a number of questions as shown in Figure 2. That is, when your answer meets all criteria of detection, response will then show up. The responses are video formats so as to arise interest and attention of the young people.

Drugout is an iOS Apps aims to passing knowledge about antidrug [8]. In the application, players will try to help a game avatar to fight with his drug abuse issues as shown in Figure 3. Score will be accumulated during the actions, and players can get bonus score if they invite others playing the application. Finally, there will be www.astesj.com real prizes given to the high score players. Overall, it is an interesting game that can attract students to play and be able to deliver the anti-drug information to the players. The bonus scoring can encourage players to invite more people play the game and players can experience what a drug abuser will suffer from the drugs. Yet, it only provides information without any communication platform. Also, its use is for the youth and parent may not get help from this application.

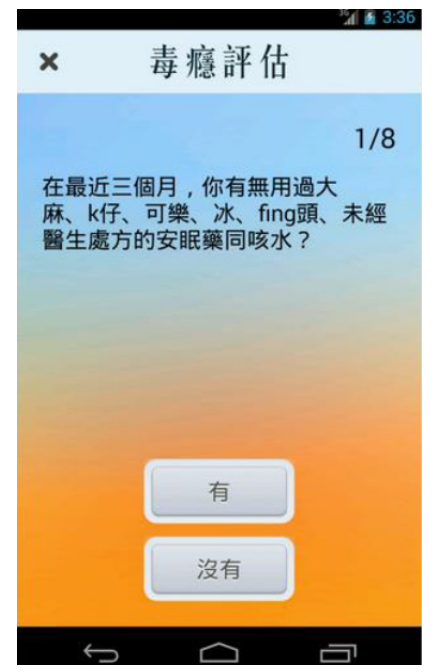

Figure 2. Addiction testing

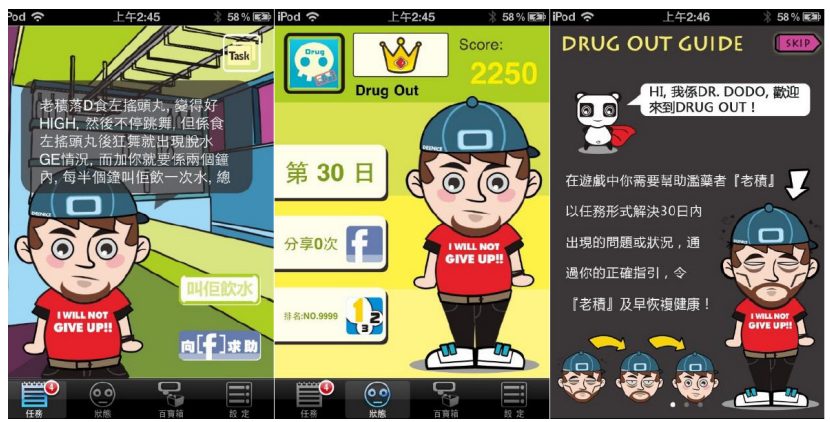

Figure 3. Dragout

Another similar mobile application is called Detoxification [9] Besides anti-drug information, it also provides interesting game and situational questions which raise awareness of drug abuse as shown in Figure 4. Yet, the detoxification application has not been completed and it also lacks of resource links to support youths when they encounter a problem.

Another type of mobile applications is for behavioral analysis, such as the "Pro-sociality Behavior Test". It contains a selfassessment to test pro-sociality behavior tendency. Its problem is having too many questions for an assessment and users often feel tired when doing it. There is a similar mobile application, "Depression Test", for depression testing [10]. It provides a nice historical review and users can see the changes of their depression levels. The application provides no connection between users and social service organizations. Users cannot find an easy way to seek for help if they encounter a problem.

Although there are many mobile applications about behavior analysis in the app stores, most of them are not related to anti-drug. 
This motivates us to develop a suitable solution with design objectives below:

- Provide an accurate prediction based on behavioral information provided by users.

- Able to detect potential drug abuse with a few guided questions with their answers.

- Able to provide connection and interaction channels between users and anti-drug social service organization.

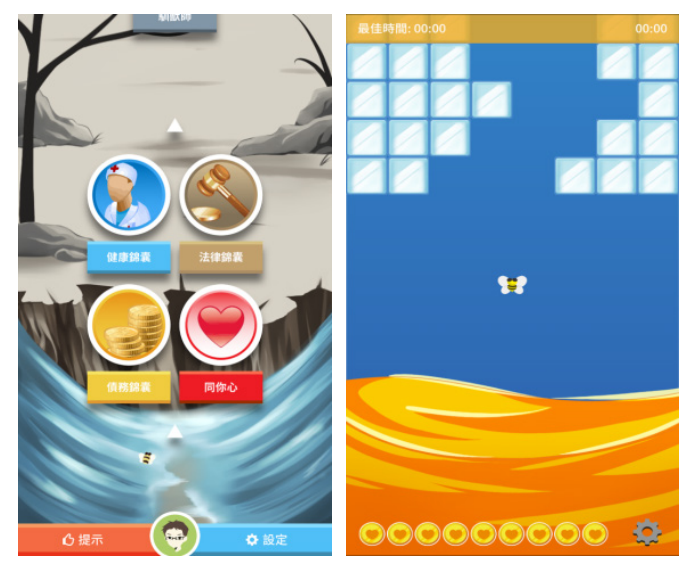

Figure 4. Detoxification

\subsection{Alerting Systems}

Alerting systems have been applied in different service domains. The Brigham Integrated Computing System (BICS) provided a clinical alerting system at Brigham and Women's Hospital (BWH) since June 1994 [11]. It used an event monitor to determine whether a new clinical data warrants an alert. The notification program would send an automated e-mail message messages to a duty physician, or through other means of communication. The physician could then log on any computer to review the alert and take immediate therapeutic action. The alerting system reduced the time it took for physicians to respond to critical laboratory results. Physicians thought that the communicated information was important and the service can help clinicians to be well informed of important abnormalities in the flood of data they have continually receiving.

Alerting system is also essential for providing the location and information about vehicles to passengers, owners or users [12]. Bus transportation system has been proposed to track a large number of buses simultaneously and detects the routes and directions automatically through GPS satellite [9]. It used a webbased, mobile communication and SMS platform for communicating bus arrival times and predicted the real-time interstation speed.

Email alerting has been proposed with education, health care and other life applications. They can be used for two-way knowledge translation, which involves receiving case situation to professionals and then sending their evidence-based recommendations. In [13], it is reported that professionals were familiar with email communication. While many felt comfortable and liked using emails in their professional life, yet some others felt overwhelmed by the volume of emails they receive. The study also suggests that email alerting can contain valid and trustworthy information as an easy way to disseminate information to multiple recipients. This would give the reader an option to read emails immediately or later.

Instant messaging alerting is another frequent channel to support alerting messages. The major advantage of instant messaging alerting is real time or instantly delivered. When a receiver is online, the message will be received as soon as the user sends it. Also, instant messaging alerting tends to be confined to a single line and easy to read [14]. However, instant messaging systems are often of proprietary platforms and cross platform support is difficult. Communicating via instant messaging requires that both parties have accounts with the same instant messaging service.

With the above reviews, we have proposed to use multiple channels for sending our altering messages, both email and instant messaging. As social workers are familiar with both channels, this multi-channel platform can effectively work for their clients.

\subsection{Location Sharing}

Check-in provides location awareness support so that it can act as a channel to share, comment and rate any spots in different areas. It aims to establish a location-based social network (LBSN) for users. All users included students, parents, social workers can publicize their current locations to others. Through this check-in step, users can contact their friends nearby and chat with instant messaging. A special function for social workers is that they can be notified the youths' current locations if youths choose to open the information.

In Google Map, users can leave comments and rate places. The comments and ratings are public to everyone in the world. More than comments and rating, users can also upload and share photos about the spot. Sharing the location with user's friends through other applications like Facebook and Whatsapp, is available in Google Map [15]. A good example is an application developed by Apple Inc., FindMyFriends. It allows users to follow and track friends with their mobile device. Users can also choose to share their location with others. The app determines users' location using GPS in the device. If the user is requested by his friends to see their current location, users can contact their friends on the map immediately with the contacts in their device [16].

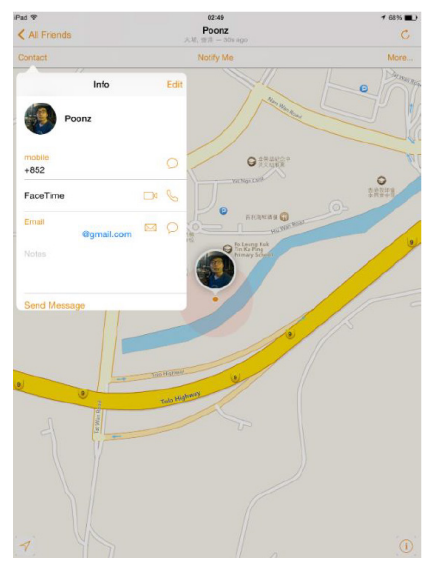

Figure 5. FindMyFriends 
Another similar application is Swarm which is developed by Foursquare. It is a companion application of Foursquare. Users are allowed to share their locations within their social network. A check-in can be broadcasted to through other channels like Facebook or Twitter. User can easily see nearby friends, make future plans as well as checking in a location. Figure 6 shows a check-in page of Swarm.

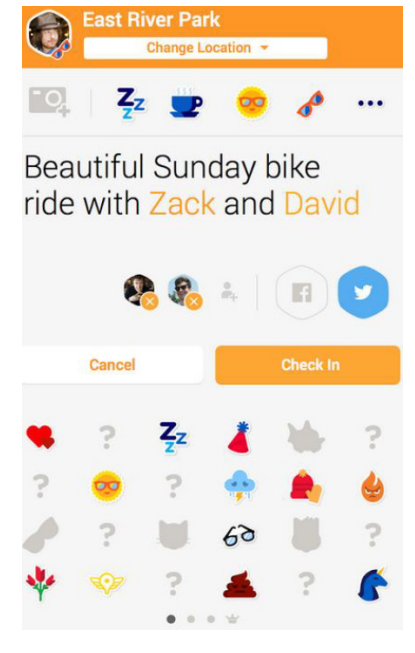

Figure 6. Swarm in Foursquare

Swarm has all the required functions for our objectives in the aspect of location sharing. From "check-in" and "check-out" to chat with friends. However, because our target users require a high level of confidentiality, only secured sharing platform is allowed.

\section{Design Methodology}

After the review the current problems in the previous sections, a set of mobile application is decided to develop to suit the needs from the different users. For parents, the mainly functions will help them on drug abuse detection of their kids and they can use the application to seek for professional help and build a community between other parents and social workers. For students, the application will try to collect their data that will be useful to the project team, also the application will help the youth have more connection with their parents, friends and social workers by instant messaging or share their location information. For social worker, they should able to use the application to upload the anti-drug information and give help to the youths and their parent. With the types of users, 3 different mobile applications have been designed and developed and they are called Aurora, Giai and Theoi, respectively.

In the rest of this section, the overall design and three functional components are presented. The first component is on the youth behavioral analysis for users to predict a potential drug abusing case. The second component is to support the alerting function when such case is detected. It can effectively work for their clients. The last is about the location sharing and near-by search support.

\subsection{Overall Design}

At the beginning of our work, teachers, principals, students, social workers and anti-drug experts have been consulted and surveyed on the suitable functions of the three applications. For the student mobile application, it includes the following functions:

a) Head News Center: An information receiving channels for youths to receive anti-drug information, such as events, activities. Some of the news can be in a form of alerting and pop-up such that youths would receive something interesting to consider. It is a way to encourage youths to get more positively involved in the community. An interest profile can be set by the young user for customized information.

b) Q\&A Section: a resourceful function for youths to receive answers of common questions, such as where and how they can seek help.

c) Survey (game corner): it is useful to collect youth's current status from time to time. They can provide some of their own data on a voluntarily basis with a bonus point encouragement. The bonus point scheme can be supported by small souvenirs (produced by the project). The survey is in the form of a small game and its data collected can be made available to the social workers and the project team.

d) Searching: a searching function for allowing the youths to quickly identify the information from (a), (b) or (e).

e) Dashboard: a sharing space for users to post/upload their digital products (games, videos, art works, etc.) for sharing. After attending training workshops available from the project, these products can be shared and users can rate them. After uploading, other users can access these products.

f) Quick Contact: this function supports a user to contact a social worker for assistance quickly. The user can enter free text or answer a number of pre-set questions and a social worker would be alerted and try to arrange help.

g) Instant Messaging: with different groups (among youths, parents, and social workers) formed, members within each group can do online chat and share information.

h) Check-In/Location Information: the function is to support users in contacting people nearby and using it as a social media platform for attracting youths. It allows a user to release his/her current location information to other users. It is a powerful tool so that a user can know any other users in the same area. Users can provide comments of the check-in locations. It is a voluntary basis and the access of the location information is limited to the people that the user allows. Some initial designs have been done as show in the next page.

The second application, Giai which focuses on parental support, is developed for parents with the functions below. For similar functions in Aurora, the functional descriptions are skipped.

a) Head News Centre

b) Searching

c) Dashboard

d) Quick Contact 
e) Instant Messaging

f) Friend List: a function which allows a user to add a friend or create a new user group (limited to some types of users).

g) InfoSeek: it is a searching function similar to the Location Information function in the Youth Apps

h) Family Center: this is the intelligent alerting tool. It enables parents to input their observations of their children on a regular basis. The input is semi-guided which can help parents to enter structured information with certain set of unusual behaviors. Once parents' observations are entered, the second function is to perform analysis with the knowledge base of an expert system available in the backend server. The expert system is designed and developed by the project team with the help of anti-drug experts and social workers. In case of parent users do not how to start, a series of prompts (similar to a quiz/survey) can be used to collect data. The analysis result would be in form of hints or advice to parents, suggesting where to seek help or acquire further information. This function can work with (f) above to ease the worries of parents.

i) Parental Corner: it is a special sharing space for parental groups and social workers to share information in addition to Dashboard and Head News Center. Parents/social workers can post articles or provide comments/advice of any articles. This corner can access special cases, emergency procedures and FAQs that are stored in a knowledge repository developed by antidrug experts and/or social workers. A searching feature similar to (b) is available also. This function is aimed to encourage parents to form mutual supporting groups for helping each other.

j) Referral Center: the function is acting a help-contact service which allows a parent to have an initial contact with a social worker. This is a resourceful function for youths to receive answers of common questions, such as where and how they can seek help.

The third application, Theoi, is developed for social workers with the functions below. For similar functions in the other applications, the functional descriptions are skipped.

a) Head News Center

b) Searching

c) Dashboard

d) Instant Messaging

e) Friend List

f) InfoSeek

g) P-Action+: Parents are able to request help from social workers with the altering tool, an email with test records will be generated automatically and sent through their own email accounts which they used in registration.

h) Y-Action+: It is similar to P-Action+ but the corresponding contacts are youths.

i) Enrich+: According to the feedbacks from social workers, a web base platform is developed for this function, instead of included in the apps. It allows social workers to post and share information, and update the FAQ database.

Figure 7 shows the software architecture of the system in which different modules are labeled with different border colors. Modules in the red rectangles mean these are shared by all 3 applications. Modules in blue, green and purple rectangle represent the functions only serve students, parents and social workers respectively.

\subsection{Behavior Analysis}

For the behavior analysis component, there are 4 core modules as the training, classifying, calculating and collecting. In training module, we have chosen 2 techniques, decision tree (see Figure 9) and logistic regression, to build a classifier with the given training data. The calculating module is to estimate an index for possible explanation of the recommendation. The collecting module will receive user's feedback and store them for the enhancement of our analysis (i.e. re-training with additional data). The 4 modules will work together to analyze behavior of youths as shown in Figure 7.

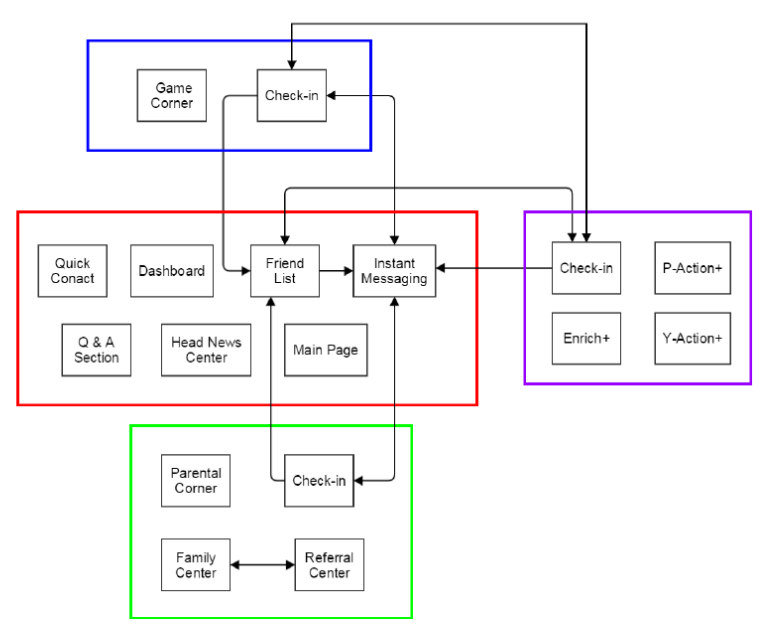

Figure 7. System modular design

The training module will run every time when the additional data is available. It will retrieve the training data and then apply a set of weight behavior criteria with the construction of a decision tree and the execution of logistic regression algorithm independently. The training results will pass to the classifying module and the calculating module afterwards. As the current available is small, the overhead of re-constructing the prediction model is acceptable. Yet, when the dataset size becomes larger, an incremental technique should be considered.

The classifying module is going to utilize the decision tree. A user is required to complete a set of guided questions which represent the behaviors of a potential drug abuser. Then the survey answers will be input to the decision tree. If it is classified as a nondrug abuser, it will bypass the calculating module and return the index as 0 . Otherwise, it will go on the calculating module.

The calculating module is going to use the results of logistic regression. It is used for selecting a explanation description by estimating an index, which refers to the probability of drug abuse. 
The determined class and index will return to the user. The collecting module is an optional module. A user can return expected status of the predicted drug abuse case to the server. This user feedback and the results of the previous survey will be added to the training data for later prediction improvement.

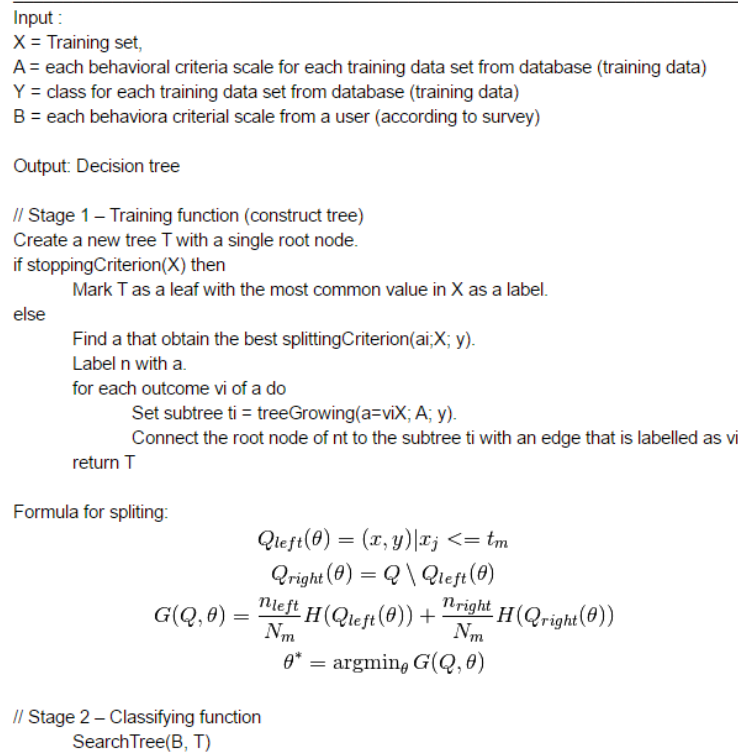

$$
\begin{aligned}
& Q_{\text {left }}(\theta)=(x, y) \mid x_{j}<=t_{m} \\
& Q_{\text {right }}(\theta)=Q \backslash Q_{\text {left }}(\theta) \\
& G(Q, \theta)=\frac{n_{\text {left }}}{N_{m}} H\left(Q_{\text {left }}(\theta)\right)+\frac{n_{\text {right }}}{N_{m}} H\left(Q_{\text {right }}(\theta)\right) \\
& \theta^{*}=\operatorname{argmin}_{\theta} G(Q, \theta)
\end{aligned}
$$

// Stage 2 - Classifying function SearchTree $(B, T)$

Figure 8. Decision Algorithm

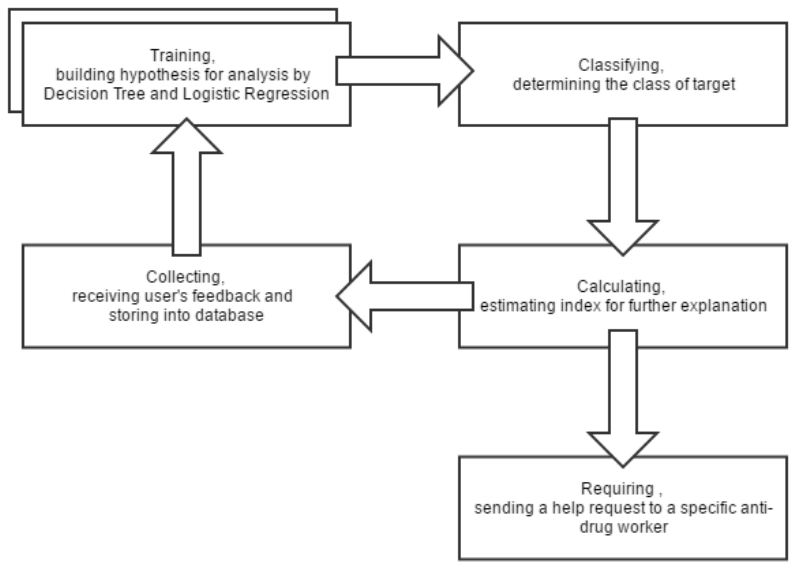

Figure 9. Behavior Analysis Framework

\subsection{Altering}

For the alerting component, there are 3 modules as confirmation, generation, and sending (as shown in Figure 10). In the confirmation module, we collect the information on the results of behavior analysis, user's demographic data, and the social worker whom users have chosen. Information would then be passed to the generation module. The generation module is used for generating email and instant message. They are generated by two servers in parallel. After that, the sending module will send the messages out.

\subsection{Location Sharing and NearBy Support}

Check-in support is a major component of our applications. Through the establishment of a healthy location-based social network (LBSN), it does not only share location-embedded information to an existing social network, but also create a new social structure by connecting individuals. With the location information, a person-to-person communication function can be enable, which is called the Friends Nearby Notification in our applications.

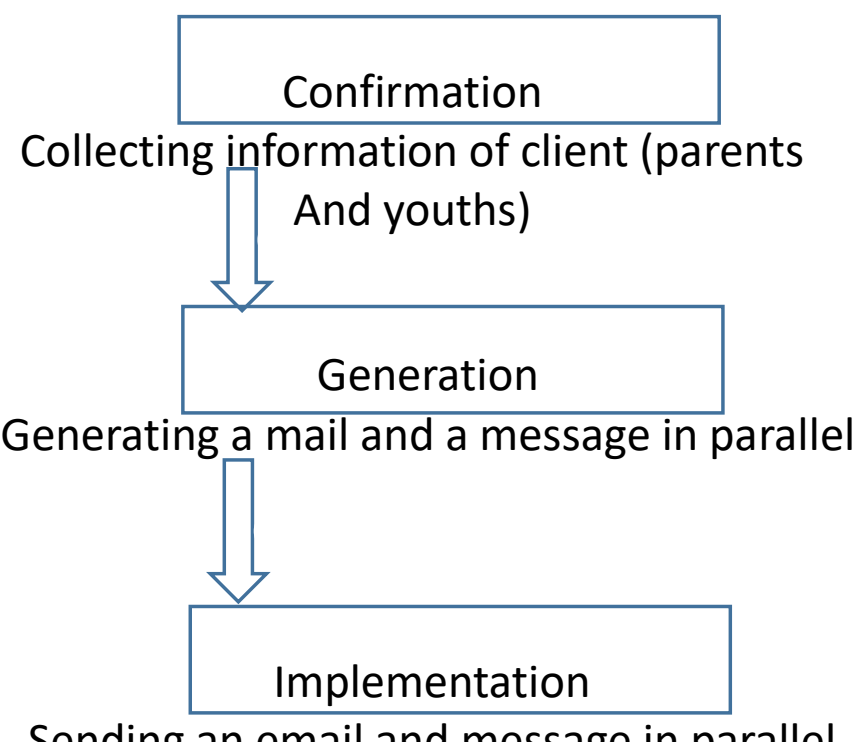

Sending an email and message in parallel

Figure 10. Alerting component

When a user would like to view others' comments to his current location, he can get them from a safe and secured database. Users can chat with their friends or other user after reading their comments. They can be redirected to Instant Messaging engine. In order to find their friends for having a face-to-face talk, the find route function is provided. The default origin will be the user's current location, and after user selecting a destination, data will be sent to the server. A string in JSON format will be returned and transformed into a polyline shown on the map which represents the route between two places. In our design, the Friends Nearby helps users to identify all nearby friends and accessibilities with the following features:

- Notify users when friends are in proximity

- Check nearby friends or accessibilities in regular time intervals

- Provide setting to turn off notification to support privacy

- Provide setting to hide from a particular friend.

- Show the nearby friends and accessibilities details, such as distance away from it, and contact info. 
- Call or send short messages to nearby friends.

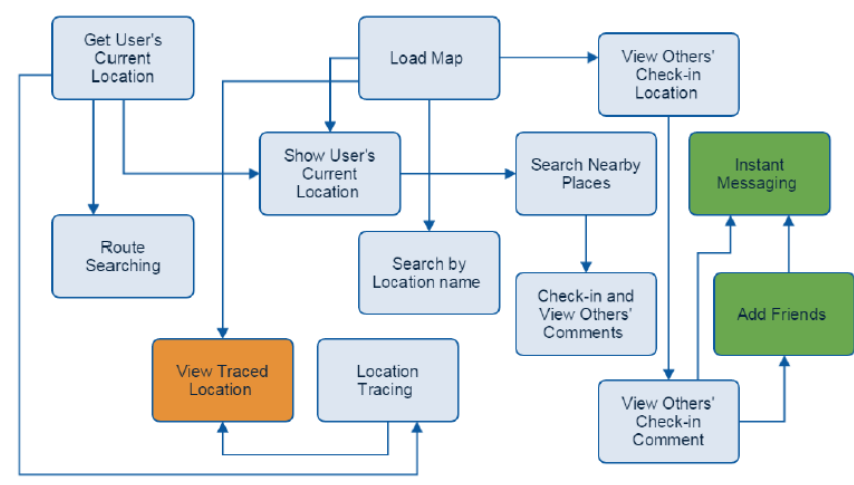

Figure 11. Check-in function

Figure 11 shows how the check-in function is designed and the starting module is the top left box in the figure, 'Get User Current Location.' The boxes in green are the functions that linked with other parts of the platform while the orange box is the function only available for social workers to trace locations of young people, provided with their permission. The locations of these young people will be uploaded to a central database for every 10 minutes. Social workers can view the students' location on the map by retrieving location information from the database.

\section{Implementation}

The mobile applications and its backend support are designed as a 3-tier client server model as shown in Figure 12. When a user uses a smartphone to access the system, requests will be sent to middle tier, i.e. web server, through HTTP request first. Then, the web server will translate the request into SQL queries and sent it to a database server to retrieve data. The return data will be transformed into JSON response and sent to the client device. All functions are based on different modules as shown in Figure 12.

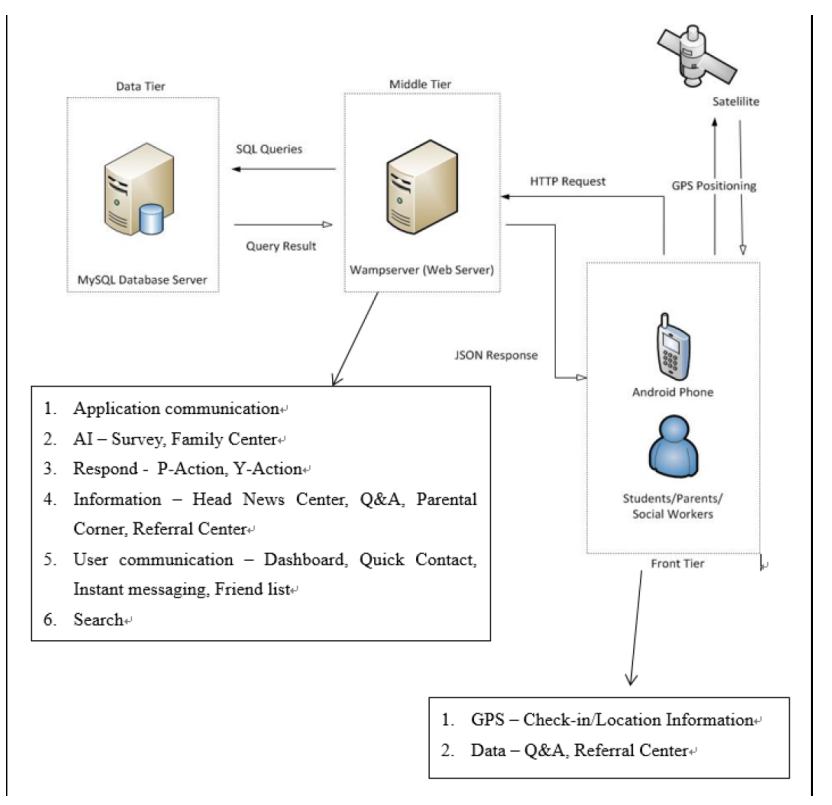

Figure12. Three tier implementation
In the current design, check-in requires only 2 relational tables. The first one stores basic user information, latitude and longitude, which are automatically uploaded by the application. The later one stores all check-in records. We do not archive all user location information since the amount of data is too large to be stored locally. For finding out a user's location, there are two common methods GPS and A-GPS. After balancing the advantages and disadvantages, we are using GPS to locate a user if it is available. If not, A-GPS will be applied. Figure 13 shows a location detection of a user.

Without GPS assistance, a user can check-in manually by entering his nearby location. Google Places API Web Service is used here since it can save device's resources. Several parameters including location, radius and API Access Key are required to retrieve the nearby locations. Location information in the format of latitude and longitude of user's current location can be gotten automatically by the system. Radius means the distance in meters within the returned place results. Check-in sets it as 100 meters since the known maximum error of A-GPS is 100 meters. After preparing these parameters properly, an HTTP request will be sent to Google. Maximum 20 locations will be returned by Google in JSON format. The returned results will contain sort of information about the location. Only place name will be shown to users in a list as shown in Figure 14.

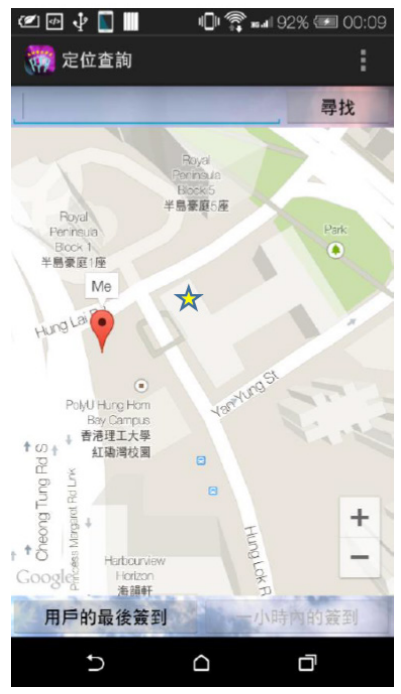

Figure13. Location detection - Check in

Check-in provides location awareness support so that it can act as a channel to share, comment and rate any spots in different areas. Users can share their current location with others. Only 4 data values are required to "check-in", i.e. username, check-in's location, comment and rating. Username will automatically be gotten by the system. Check-in location is stored after user finds the nearby locations. A user can also enter comments and ratings about the location in Check-in page as shown in Figure 15. An error will be shown if the length of a comment is more than 160 English characters and 80 Chinese characters. A limit is set with the same idea from the famous social network like Twitter and Weibo. They have a special feature that the post length is limited. According to [17], 160 characters provide plenty of space to express most of their thoughts with friends. Chinese messages can usually present more information or feelings with fewer words. 
Thus, 80 characters should be a proper length constrain in a checkin comment.

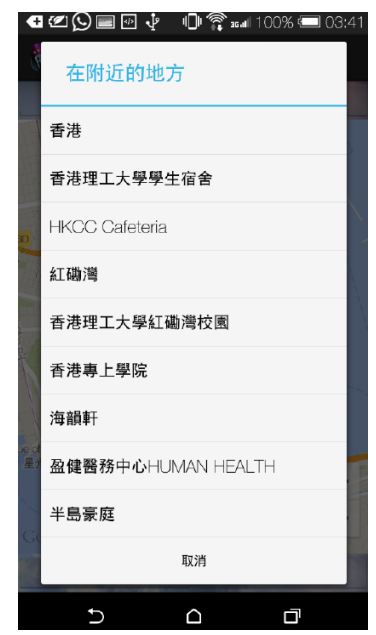

Figure14. A list of NearBy locations is returned

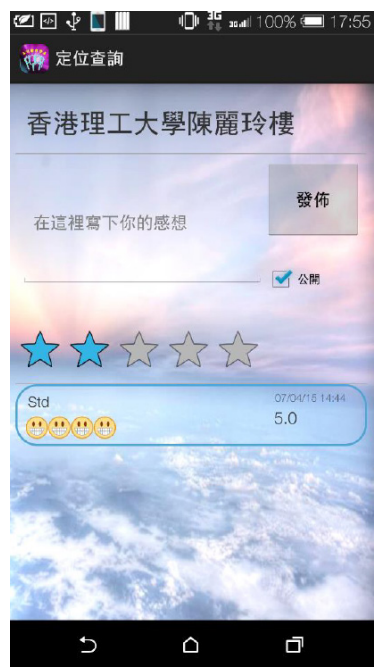

Figure15. Comments for a checked location

The behavior analysis module is implemented with Python 2.7 at the server end. We have selected the scikit-learn toolkit to support the decision tree and regression analysis [18]. The CART (Classification and Regression Trees) is used for building a model to determine if a person is a potential drug abuser. The same toolkit contains the logistic regression function in its 'liblinear' library and it is adopted.

The Drug Abuse Screening Test (DAST-10) is a 10-item brief screening tool formed the basis of the data being used in the behaviors modules [19]. Other data attributes, such as sleeping patterns, school academic performance, washroom visiting frequency and periods being alone, are included. During of the development of the module, the project team could not acquire the live data because of privacy issues. Instead, a pseudo dataset of 250 records (cases) is generated randomly with 20 attributes per case. Anti-drug experts were then invited to label the cases with different level of drug abuse (where 0 means nil and 5 means high potential). The first 200 records have been used for the decision tree and regression analysis in the scikit-learn toolkit. Cross- validation was then used to verify the accuracy and the results were in the range of $70-80 \%$. The last 50 records were used as additional data for the continuous training. Yet, the results have not been improved.

The email alerting method is mainly based on PHPMailer. It is an open source class with methods to support HTML-based email and attachments. The messaging alerting method is mainly based on Messaging Queuing Telemetry Transport (MQTT) [20, 21]. MQTT is standardized by OASIS as standards to transport data. In MQTT, there is no message formats specialized in it and standard used is depending on developers. But it has advantages over using HTTP and XMPP, which it is low-weight in transmission as lower size in overhead and more energy saving [22,23].

With the above set up environment, three mobile applications have been developed. The mobile apps for parents contain the alerting tool. It has a main page which displays Drug Abuse Prediction and or Result Feedback (see Figure 16).

In the behavior analysis, the parents act as observers to record their children's behaviors. When the parent starts the drug abuse prediction test, a series of questions on youths' behavior has to be answered in order to get the prediction results. The estimated probability of drug abuse is returned to the screen of the mobile device as shown in Figure 16. When a warning or alerting message is received, parents can seek help from social workers who have joined the platform according to their regions. In seeking the help, parents can provide more specific information about themselves and their children so that suitable social workers in the district can be located faster. They can also describe the situation in order to shorten the remedial time. When a help request is sent, the selected social workers will receive a notification from our platform as a reminder and email message describing parent's situation.

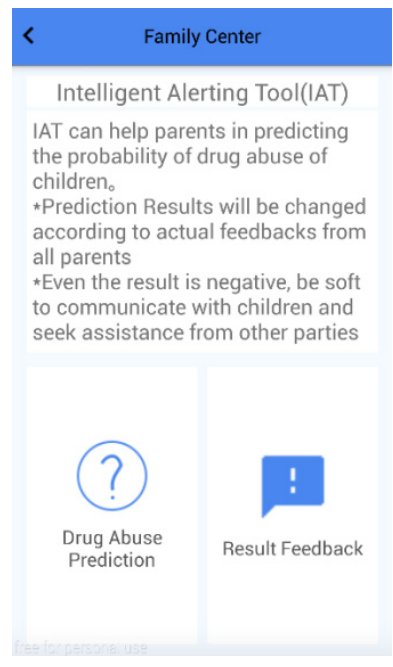

Figure 16a Main Page of behavior analysis

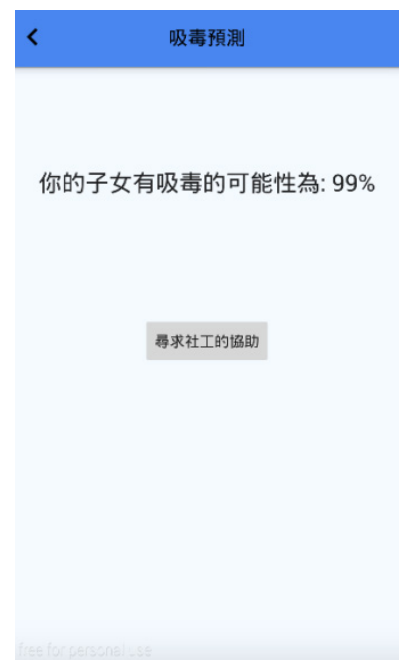

Figure $16 \mathrm{~b}$ Result of probability of drug abuse
For result feedback, parents are required to answer "positive result" or "negative result" to confirm the drug abuse problem of their children (see Figure 17b). And the feedback is limited to previous detection, which any new prediction will overwrite the old predictions for feedback. 


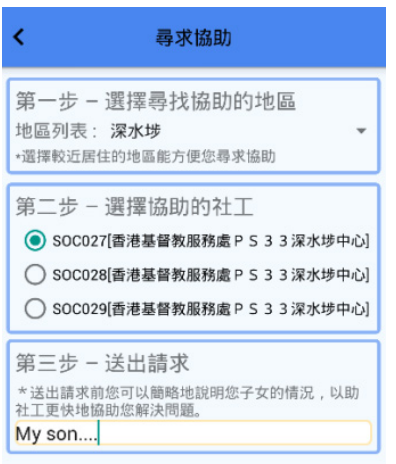

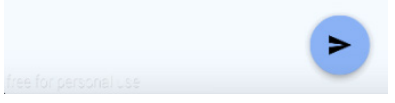

Figure 17a Seek help from social worker in different district

\section{Conclusions}

In this paper, we have presented three mobile applications with a set of intelligent tools for parents and social workers to help our young people as shown in Figure 18. The application for parents has a tool to analyze drug abuser behavior and send alert to them. The applications have been placed in GoogleStore for download availability.

The work is funded by the Beat Drugs Fund of HKSAR. We have invited a number of secondary schools, community centers, anti-drug experts and social workers to try the applications. All data is collected with a centralized server with confidentiality protection and agreement from users. Users can have options to select if any personal data can be used. Towards the end of the project, positive feedbacks from different user groups have been received. Many parents are interested in the alerting tool while expecting more rules and advice can be embedded.
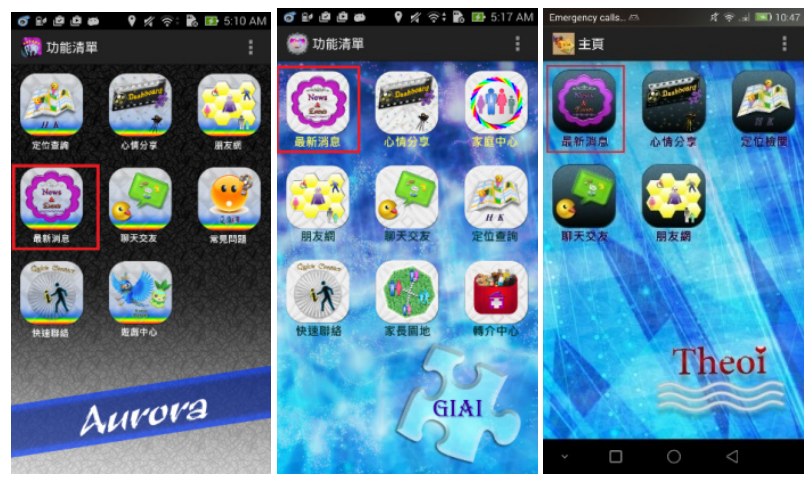

Figure18. Three mobile applications for anti-drug abuse

\section{Conflict of Interest}

The authors declare no conflict of interest.

\section{References}

[1] A. Isobe, 'On publication of denominational precious volumes (Jiao pai xi Pao juan) in Late Ming and Early Ch'ing as seen in, "Niwatazumi".', Studies of Publishing Culture in East Asia, vol. 8, pp. 187-226, 2004.

[2] H. Chan and T. Lo, 'Quality of Life of the Hidden Youth in Hong Kong', Applied Research Quality Life, vol. 9, no. 4, pp. 951-969, 2013.

[3] The Central Registry of Drug Abuse, "Central Registry of Drug Abuse Sixtysixth Report," Narcotics Division, Hong Kong, 2016.

[4] Policy 21 Limited, "A review of estimation methods on prevalence of drug abuse Final Report," Narcotics Division, 2013.

[5] Social Surveys Section, "Thematic Household Survey Report No. 54 Information technology usage and penetration," Census and Statistics Department HKSAR, 2015.

[6] 禁 毒 資 訊 站 [Online]. Available: https://apkpure.com/\%E7\%A6\%81\%E6\%AF\%92\%E8\%B3\%87\%E8\%A8\% $8 \mathrm{~A} \% \mathrm{E} 7 \% \mathrm{AB} \% 99 /$ com.antidrugs

[7] 許寶強, "校園驗毒：「認真」調查結果 馬虎禁毒措施," 明報, 2010 .

[8] DrugOut [Online]. https://itunes.apple.com/ao/app/dugout/id1163815036?mt=8

Available:

[9] Dihua Sun, Hong Luo, Liping Fu, Weining Liu, Xiaoyong Liao, and Min Zhao, "Predicting Bus Arrival Time on the Basis of Global Positioning System Data", Transportation Research Record: Journal of the Transportation Research Board,No. 2034, D.C., 2007, pp. 62-72.

[10] Depression Test [Online]. Available: https://www.psychologytoday.com/tests/health/depression-test

[11] " Cloud Messaging|Google Developers," Google Developers, 2015. [Online]. Available: https://developers.google.com/cloud-messaging/?hl=zh-tw.

[12] "Messenger for Android - Documentation - Facebook for Developers," Facebook Developers, [Online]. Available: https://developers.facebook.com/docs/messenger/android.

[13] Hani Badran, Pierre Pluye, Roland Grad, "Advantages and Disadvantages of Educational Email Alerts for Family Physicians", J Med Internet Res 2015;17(2):e49.

[14] BradleyTony. (2012). Email vs. IM vs. SMS: Choosing the Right One. PCWorld.

[15] Google Maps will let you share your location with friends and family for a specific period of time [Online]. Available:,https://techcrunch.com/2017/03/22/google-maps-now-lets-youshare-your-location-with-friends-and-family-for-a-specific-period-of-time/

[16] "Google Map," Google, [Online]. Available: https://www.google.com.hk/maps?source=tldsi\&hl=zh-TW.

[17] "Find My Friends," Apple Inc., [Online]. Available: https://www.apple.com/apps/find-my-friends/.

[18] Scikit learn [Online]. Available: http://scikit-learn.org/stable/

[19] The Drug Abuse Screening Test (DAST-10) [Online]. Available: https://www.drugabuse.gov/sites/default/files/dast-10.pdf

[20] "MQTT," Mqtt.org, 2015. [Online]. Available: http://mqtt.org.

[21] Y. Zhu, "Mobile Application Message Push and MQTT Protocol," in Wireless Internet Technology, vol. 8, 2015, pp. 1-3.

[22] "The XMPP Standards Foundation," Xmpp.org, 2015. [Online]. Available: http://xmpp.org.

[23] W. Huang, "Design of Android Instant Messaging system based on XMPP agreement," in International Electronic Elements, vol. 19, no. 8, 2011, pp. 5759. 